\author{
دراسة تصنيفية مظهرية مقارنة لنوعين من انواع الجنس \\ Chaenorhinum (D.C) Reichb. (Scrophulariaceae) \\ النامية بريا في العراق. \\ علي هاثم الموسوي ****

 \\ فاتن حسبن الدلييسيث

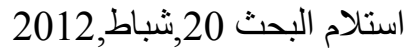

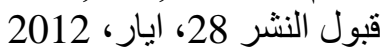

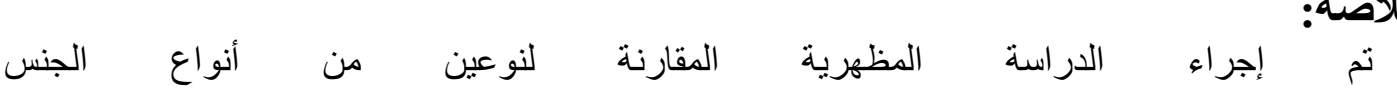
Chaenorhinum (D.C.) Reichb

1. Chaenorhinum calycinum (D.C) Reichb.

2. Chaenorhinum rubrifolium (Robill. \& Cast. Ex Lam. \& DC.) Fourr

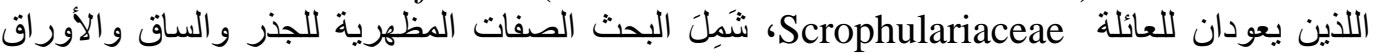

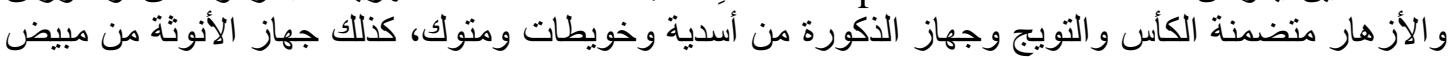

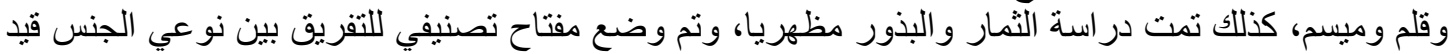

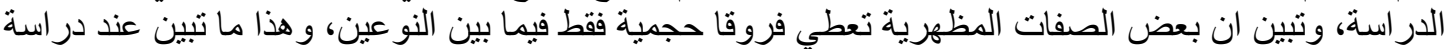

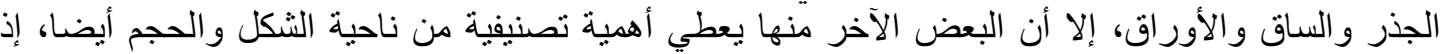
أوضحت الدر اسة وجود فروقات الأن التنيفية واضحة في أنثكال الثمار و البذور.

الكلمات المفتاحية: C.rubrifolium , C.calycinum ، العائلة Scrophulariaceae ، دراسة

أما معاملة [5] للعائلة فنتقق تقريبا مع [3] [4] من

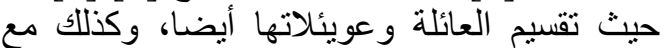
[6] و [7] و [8]. علما أن [9] أثشار الى أن للعائلة

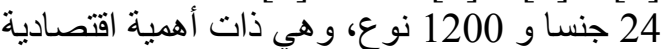
قليلة، وبعض أنو اعها نزرع ونو بوصفها نباتات زينة.

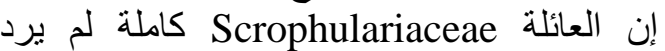

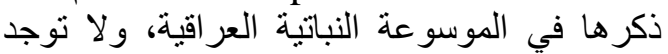

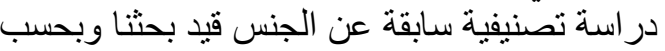

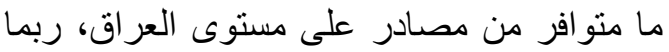
بسبب قلة انتشاره أو بسبب صني صغر حجم النباد النبات ككل.


بالجنس قبد دراستنا، لان كل ما هو متوافي افر عن من


موجود في قو ائم ذكرت من باحثين سابقين، كما في

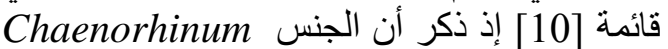

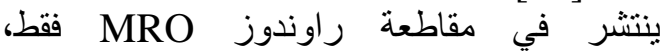
وأوضحت هذه القائمة وبشكل جزئي وارني وجود هذا



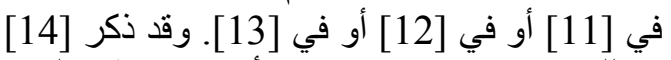
إن للجنس 20 نوعا تنتشر في أوربا ومنطقة البحر الأبيض المتوسط وغرب آسيا. وينتشر نوع نوع آخر الخر C.minus (L.) من أنواع الجنس في بريطانياو Lange.
المقدمة:ة

تعد الدراسات المظهرية هي الأساس غالبات في الأني

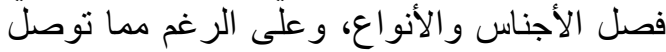

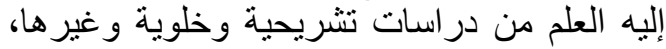

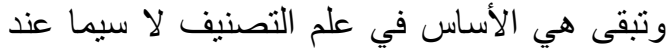

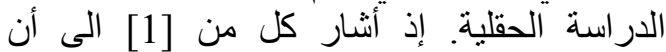
الخصائص المظهرية تتمايز بسهولة ملإية ملاحنتها

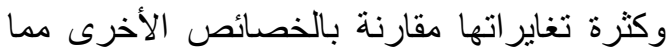

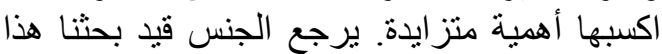

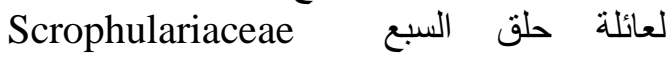
والمشهورة بإسم (Snapdragon Family) التي التي تعد من العائلات الكبيرة إذ أنها تضم 210 جنس و

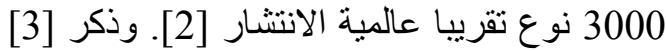

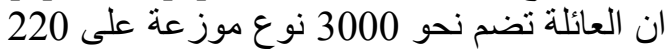

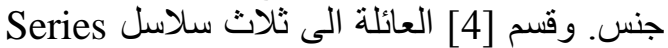

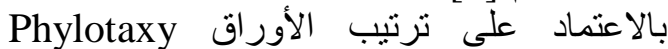

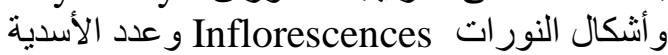
Stamens وشكل التويج Corolla وهي:

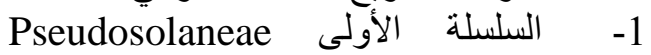
وتضم ثلاث عثائر الأل 2ست عشائر.

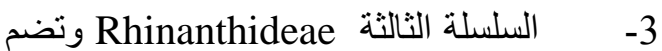

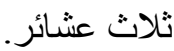


ومعشب متحف التاريخ الطبيعي ومعشب كلية

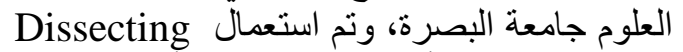
Microscope

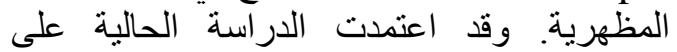

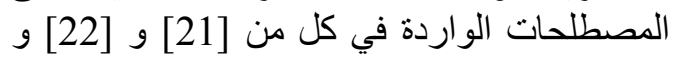

]23] و [24]

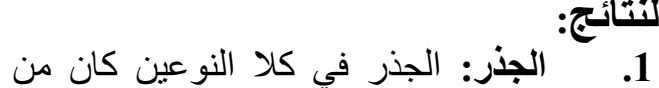

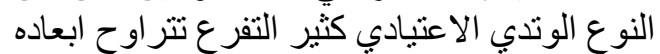
في النوع C.calycinum بنحو (20-50) (4-2) ملم وفي النوع C.2ubrifolium بنحو

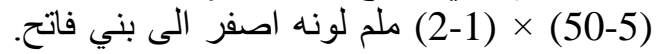

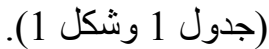

2. لون اخضر فاتح عديمة الثُعيرات في النوانية أنوانية C.calycinum

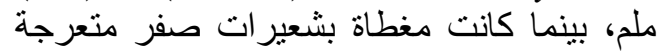

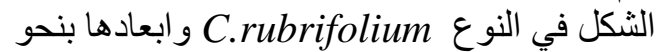

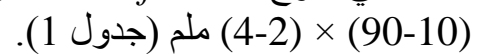
3.

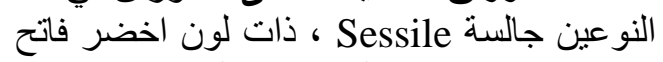

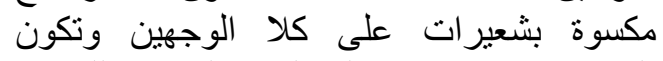
الثعبرات اكثر كثافة على الوجه الظهري للون الورقة.


، Oتصالبة متقابلة Opposite decussate رمية عريضة الى الهية اليجية Elliptic lanceolate كاملة، قممها مدبية، ابعادها (5-30) ×

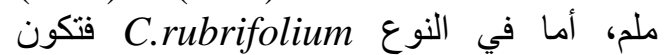

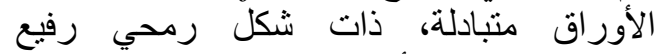
كال Lanceolate ، وأيضا قوات اعدها مثلثة، وحو افها كاملة، وقممها مدببة، أما أبعادها فتكون أنكون بنحو



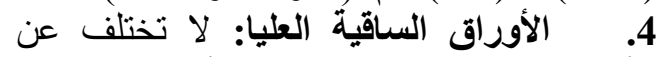





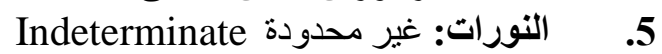
Simple من النوع العنقودية البسيطة racemose

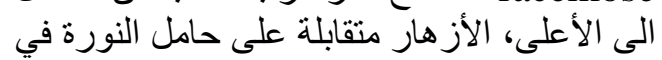

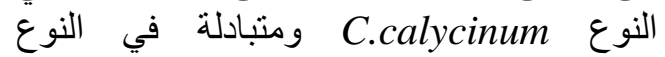

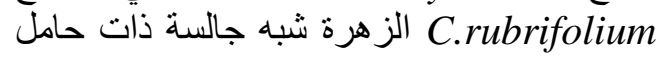
اسطو اني طوله بنحو (1-2) ملم.

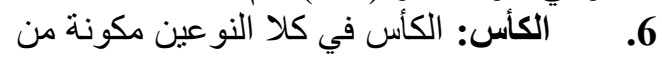

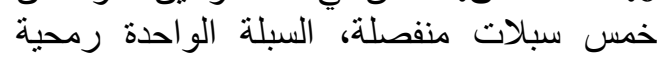
الثكل مدببة النهاية مكسوة من كلفية كلا الوجهين بشعيرات بيض طويلة، أبعاد الكأس في النوانة

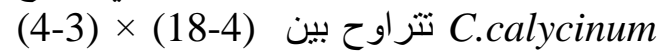

استراليا [16]. و واعتمدت الدراسة بدرجة كبيرة





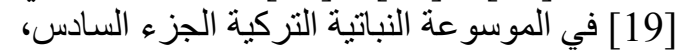
إن الجنس Chaenorhinum ينتشر في سوريا النسيا

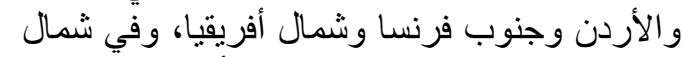

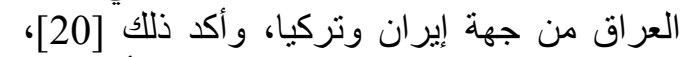
وذكر انتشار هذا الجنس في إيران وذيا، وأنكر أن الاسم


Linaria rubrifolia o rubrifolium ع Robill. \& Cast. Chaenorhinum calycinum فالاسم القديم هو . Antirrhinum calycinum انتشار الجنس في العراق:

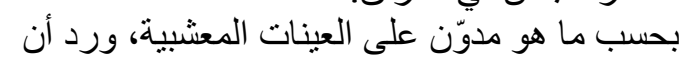

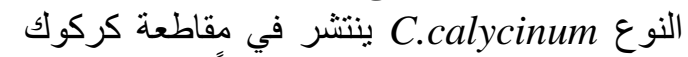
(FKI) خورماتو بمحآذات الحقول المستزرعة فئ، ويوند

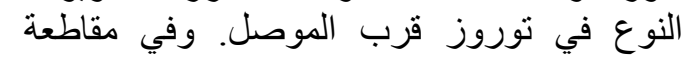
المرتفعات الثرقية الحدودية (FPF) في خدي خانقين

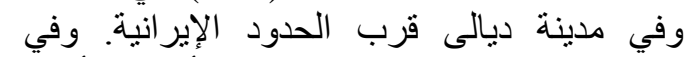

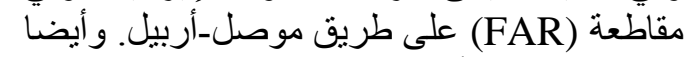

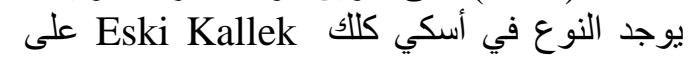
حافات الحقول. وفي مقاطعة الصحر اءك الغربية ينتشر النوع على بلى بعد 151 كيلومنر (DWD) شمال شرق الرطبة بالقرب من كرمة ام راشي الثد في

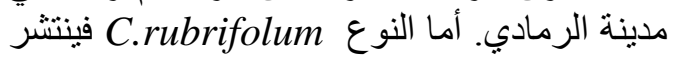


وفي مقاطعة أربيل (FAR) قرب جسر هوبة هوبكن

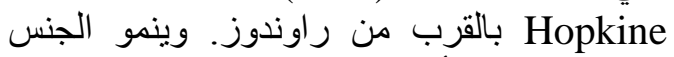

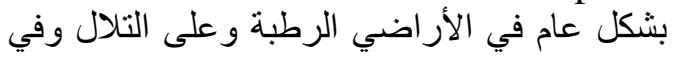
جوانب الحقول. إن الهدف من البحث هو الرئ دراسة تصنيفية للجنس Chaenorhinum من الناحية النية النية

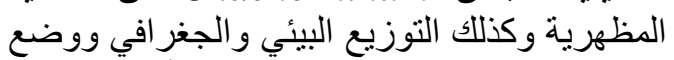

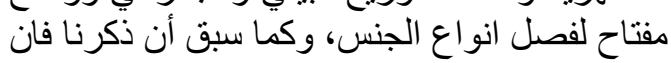

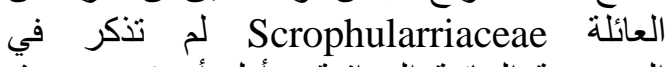
الموسوعة النباتية العراقية ونأمل أن تضيف هذه

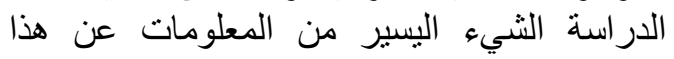
الجنس.

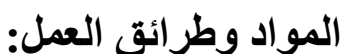

تم الاعتماد في هذه الدراسة على العى العينات المودعة

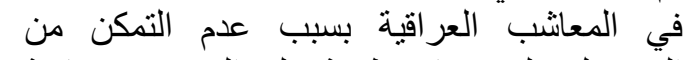
الحصول على عينات طرية على الر بلى الرغم من متابعة

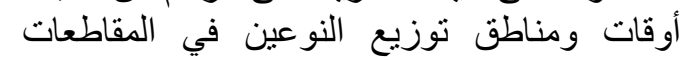

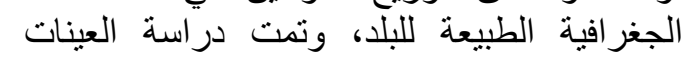

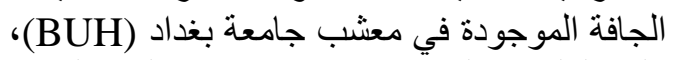

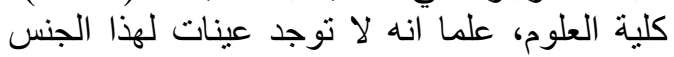
في اغلب المعاثب التي تمت زئب زيارتها في بغداد وشمال العراق وجنوبه، مثل معشب كلية الزّر اعة لغئ 
الاقرب للقاعدة بمسافة (1-2) ملم، اطوال

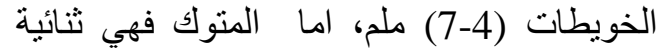
الفص وذات شكل بيضي ذي نهاية مدبية يمكن ان

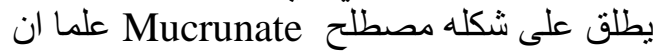
هذا المصطلح بطلق على قمة الورقة ذات المات النهاية

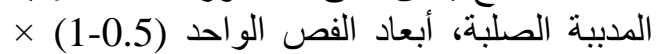
كل (0.5) ملم، وتتفتح بوساطة شق المق طولي على طول كل فص من فصي المناك.

9. المبيض والقلم والميسم: في النوع التون

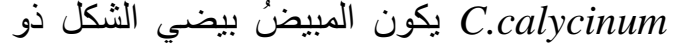

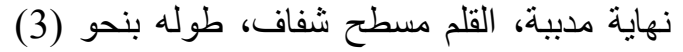
ملم، اما في النوع C.rubrifolium فيكة فيكون المبيض كروياً تقريباً ذو نهاية النواية مدبية، القلم



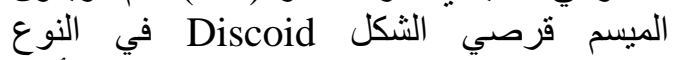



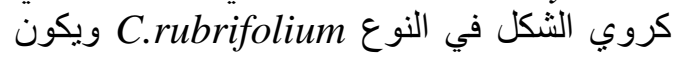
حليمي اي انه تبرز من سطوحه حليمات صغيرة.

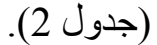

ملم، وفي النوع C.rubrifolium بنحو (3-3) × (4-3) ملث.

7.

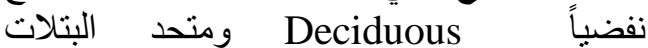

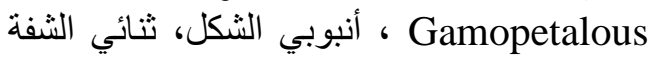

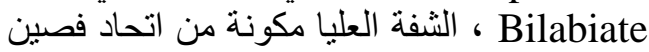

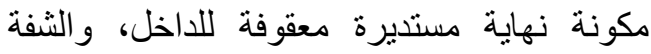

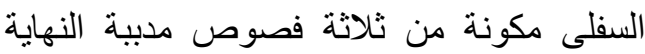
ابعادها في النوع C.calycinum بنحو (5-10)

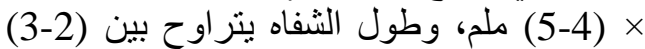

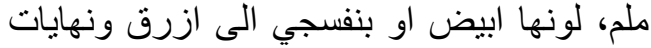

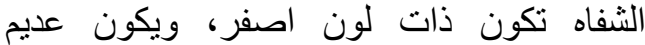

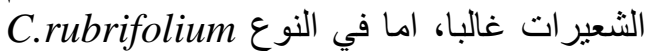

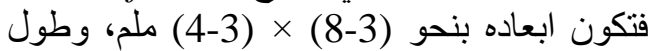

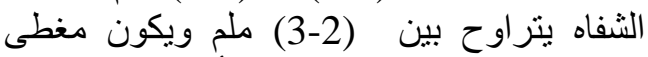

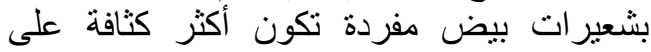
الوجه الظهري للتويج. (جدول 2)

8 أربع، طويلة الاثنتين Didynamous تكون الإني الخويطات متحدة مع التويج في الربع الاول

جدول (1) صفات الجذر والساق والأوراق للنوعين قيد الدراسة.

\begin{tabular}{|c|c|c|c|c|c|}
\hline \multicolumn{2}{|c|}{ الورقة } & \multicolumn{2}{|c|}{ الساق } & \multirow[b]{2}{*}{ أبعاد الجذر / ملم } & \multirow[b]{2}{*}{ النوع } \\
\hline أبعاد الورقة/ & الثكل & السطحي & أبعاد الساق/ ملم & & \\
\hline $10-2 \times 30-5$ & رلى الهية عريضة اليجية/ & الشعير ات عديمة & $\begin{array}{c}\times 150-35 \\
6-2\end{array}$ & $4-2 \times 50-20$ & C.calycinum \\
\hline $3-2 \times 10-5$ & رمحية/ متبادلة & بالشعبرات & $\begin{array}{c}4-\times 90-10 \\
2\end{array}$ & $2-1 \times 50-5$ & C.rubrifolium \\
\hline
\end{tabular}

جدول (2) صفات الكأس والتويج والقلم والميسم للنو عين قيد الدراسة

\begin{tabular}{|c|c|c|c|c|c|c|c|}
\hline شيسل & ملم القلم/ & شكل القلم & السطحي الكساء & التويج/ ملم & السطحي الكساء & أبعاد الكأس/ ملم & النوع ل \\
\hline قرصي & 3 & شفافح & الثعيرات & $\begin{array}{c}\times 10-5 \\
5-4\end{array}$ &  & $\begin{array}{c}\times 18-4 \\
4-3\end{array}$ & C.calycinum \\
\hline كروي & $2-1$ & اسطو اني & بالشعير ات & $\begin{array}{c}\times 8-3 \\
4-3\end{array}$ & الشعير آت & $\begin{array}{c}\times 5-3 \\
4-3\end{array}$ & C.rubrifolium \\
\hline
\end{tabular}


جدول (3) صفات الثمار والبذور للنوعين قيد الدراسة.

\begin{tabular}{|c|c|c|c|c|}
\hline أعدادها & شكل البذور & أبعادها/ ملم & شكل الثمرة & النوع \\
\hline $200-80$ & رباعي الأوجه ذو & $5 \times 5$ & 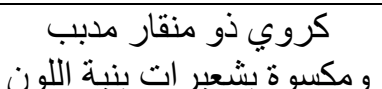 & C.calycinum \\
\hline $38-32$ & بيضية ذات اشو الك & $2 \times 3$ & مكعبة الثكل تقريبا عديم & C.rubrifolium \\
\hline
\end{tabular}

الثكل تكون أكثر كثافة على الوجه الظهري

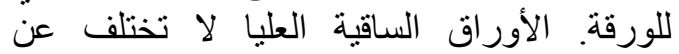

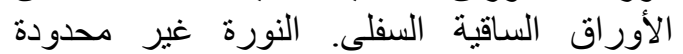
Indeterminate Simple racemose من الأسفل الى الأعلى، الأزهار متقابلة منصالبة الألية

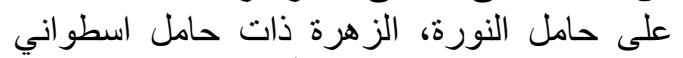

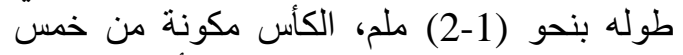

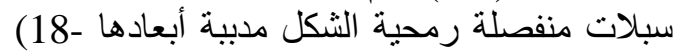

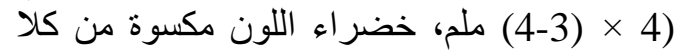

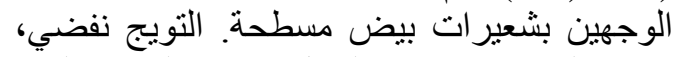
متحد البتلات انبوبي الثنكل ثنائي الثنفة، الثنفة

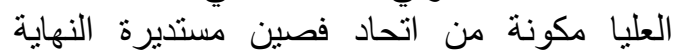

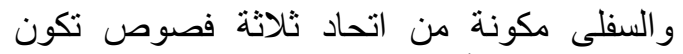

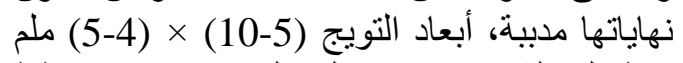

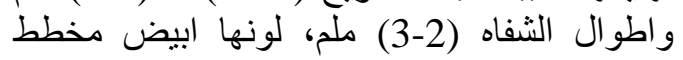
باللون البنفسجي او الازيفاه

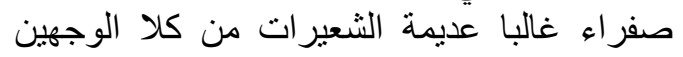

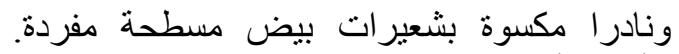



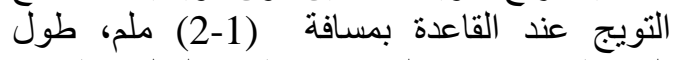

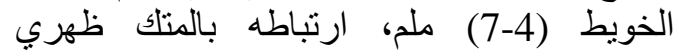

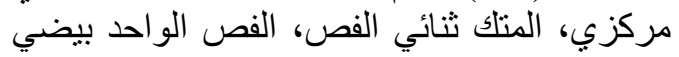
الثكل مدبب النهاية اصفر اللون ابعاده (-5.5 (-0.5-1)

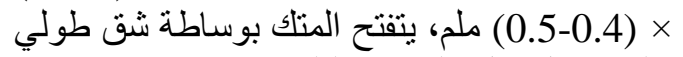

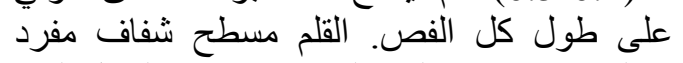

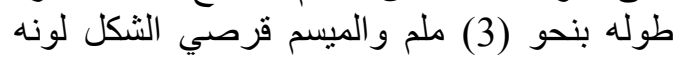

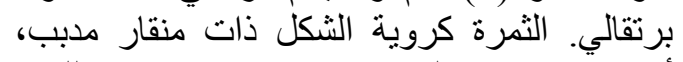

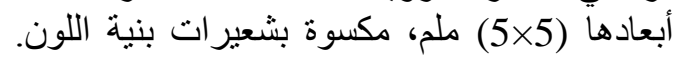
البذور رباعبة الأوجه ذات ألخادية أنسيد وحفر مشكلة

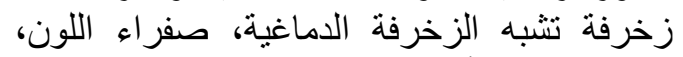
عديدة نتراوح أعدادها بين (80-200) بذرة الزفة لكل

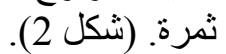

Type: (Syria) Aleppo, Solander

\section{B: Chaenorinum rubrifolum Reichb.}

عشب حولي، الجذور وتدية اعتيادية ذات

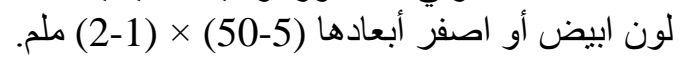
الساق اسطوانية قائمة مفردة البعادها عديمة الثعيرات،




أبعادها (5-10) × (2-3) ملم، حو افها كاملة قممها
10

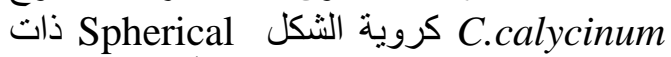



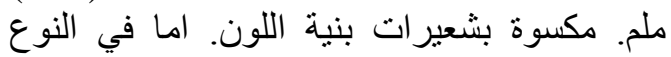
C.rubrifolium

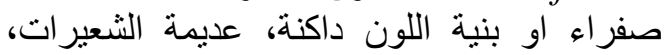

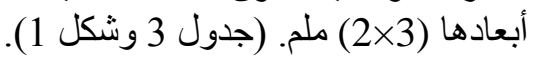

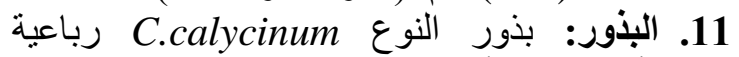
الأوجه ذات أخاديد وحفر ، صفراء اللون التون، عديدة



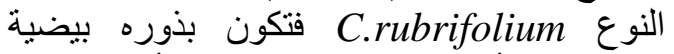

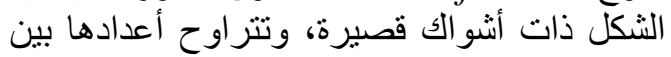

(38-32) بذرة لكل ثمرة. (جدول 3 وشكل 1ح 1).

مفتاح لفصل نوعي الجنس Chaenorhinum

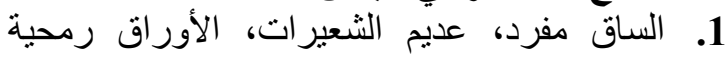

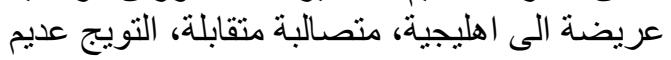
الثعبيرات، القلم مسطح الثكل، الثرة الثرة كروية الثرية

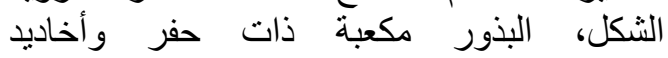
Chaenorhinum calycinum

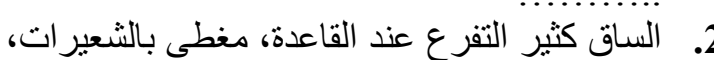

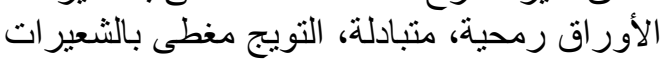
من الخارج، القلم اسطو اني اعتيادي، التوري، الثمرة مكعبة، البذور Chaenorhinum

rubrifolium.

$$
\text { الوصف العلمي للنوعين: }
$$

A: Chaenorhinum calycinum Reichb عثب حولي، الجذور وتدية اعتيادية ذات لون ابيض أو اصفر أبعادها (20-50) ×

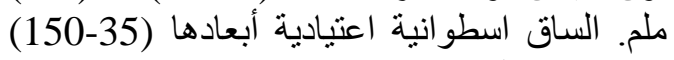



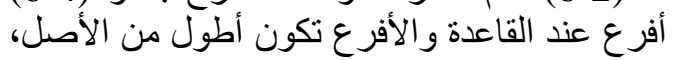

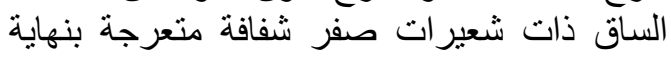

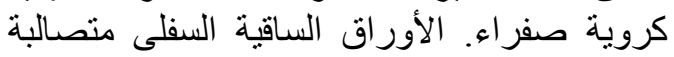

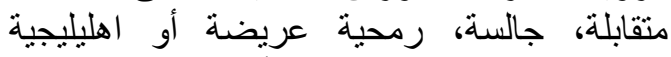

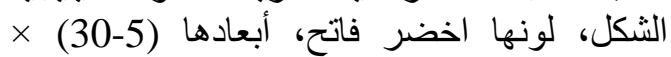

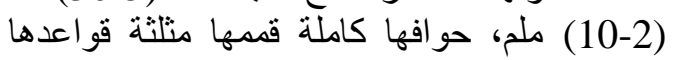
ممتدة مع الساق، مكسوة بشعيرات كثيفة متعرجة 
DWD: $151 \mathrm{~km}$. N.E. of Rutba at Kamra um Rashad, Ramadi liwa, 12-3-1962, Al-rawi, 0019035 (BUH).

FKI: $14 \mathrm{~km}$. s. of Tuz khurmato, Fallow field, 22-3-1961, Al-rawi, 0019040 (BUH).

\section{C.rubrifolium النوع: B}

DGA: Samarra, Rain field difrention, 28-5-1957, Al-rawi, 0019032 (BUH).

FAR: Hopkine bridge, Arbil liwa, near Rawandus Gorge 14-6-1960, Al-rawi, 0019034 (BUH).

\footnotetext{
المناقشة :

من خلال الدراسة الحالية تبين إن للجذر الإنائ وشكل النور ات و الكأس و الأسدية اختلافات بسنية الأنة فيما بين النوعين لا تتعدى الفروقات الكات في الأبعاد غالباً، أما صفات الكساء السطحي فكانت ذات ذات التات

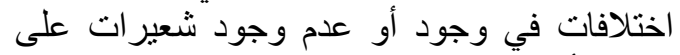
بعض أعضاء النبات في كلا النوعين، وكان إنت

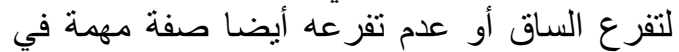



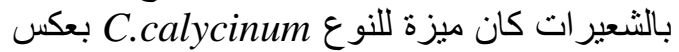

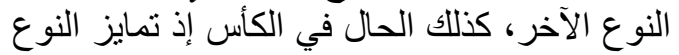




النوع C.calycinum بانعدام الثعيرات على كلاج الوجهين الخارجي والداخلي، وفي جهاز الأنوثئة الذئن كان القلم مختلف الثكل في النوعين، إذ في كان الذان مسطحا شفافا في النوع C.calycinum في حين النيان كان اسطو انيا اعتياديا في النوع الآخر، أما الأور اق فين

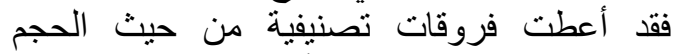

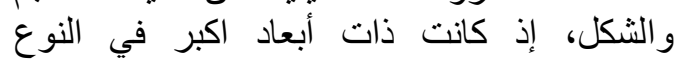

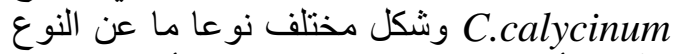

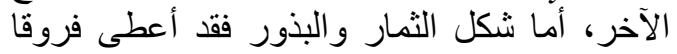

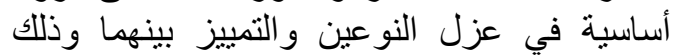

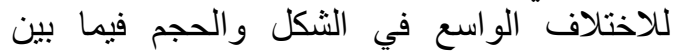

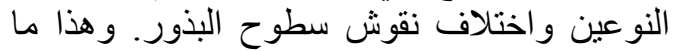


الوصف العلمي للانواع. و وعلى الرغم من إجراء الجراء



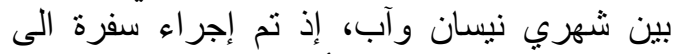

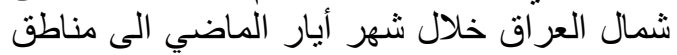

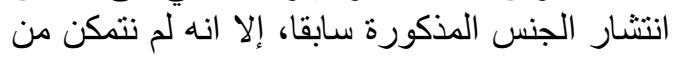

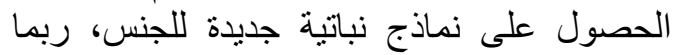

مثلثة قو اعدها ممتدة مع الساق، مكسوة من كلا الوجهين بشعيرات صوفية الثُكل بيضاء التهاء اللون

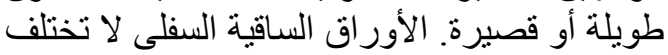

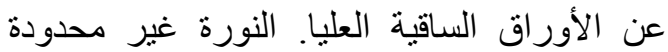
Indeterminate Simple racemose

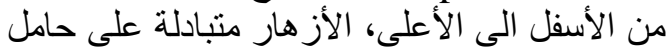

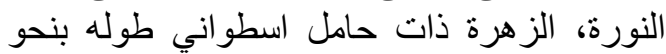

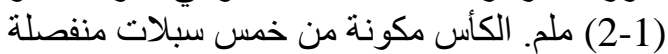

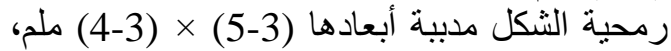

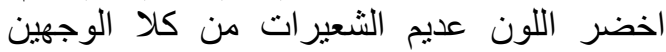

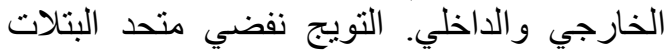
انبوبي الثنكل ثنائي الثفة، العليا مكونة من التحاد التياد

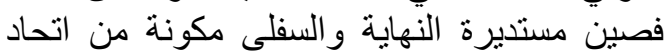
ثلاثة فصوص تكون نهاياتها مدبية، أبعاد التويج



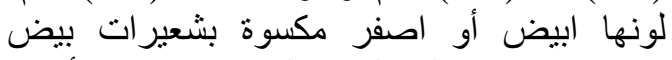

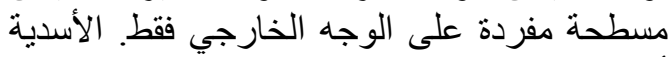

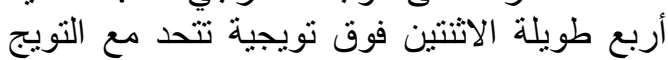



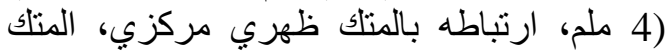

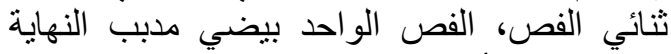

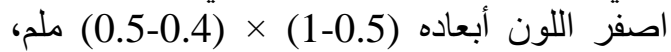
يتفتح المتلك بوساطة شق أبواد طولي على طول كل




حليمات دقيقة، برتقالي اللون. آلثمرة مكعبة الثبية الثكل

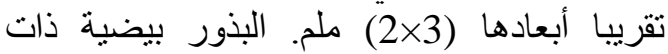

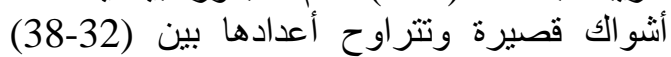

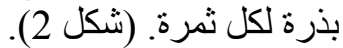

Type: S. France

البيئة: ينتشر النوعان في الأراضي الرطبة والتنال

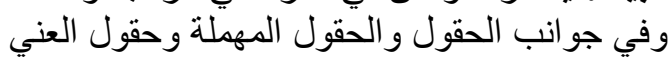

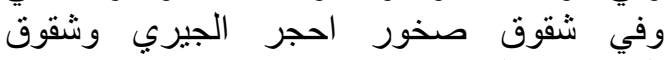
الجنران. على ارتفاع يتراوح بين (2000-350) الانتشار: ينتشر النوعان في العراق [10] و أوربا




وجنوب فرنسا وشمال أفريقيا [19] .20] [19]

\section{نماذج مختارة من العينات المدروسة: \\ C.calycinum النوع: A}

FPF: Khanagin, in Diyala liwa Hills near Persian frontier, 5-4-1957, Alrawi, 0019042 (BUH).

FAR: Khasrt bridge, Mousl - Erbil, 22-3-1961, Al-rawi, 0032424 (BUH). 
إليه إن النوع C.rubrifolium نم جمعه من الباحث Agnew في العام 1960 على انه عائد إلى دن الجنس Linaria وتمت إعادة در استه وتنتخيصه الجيه و عائديته إلى الجنس C.rubrifolium من الباحث الراوي في العام 1985 كما مثبت على العنى العينات

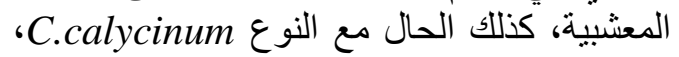

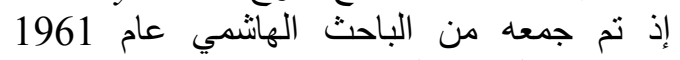

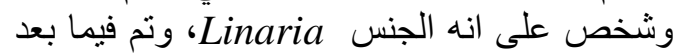

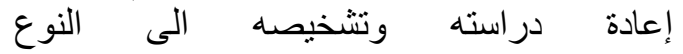

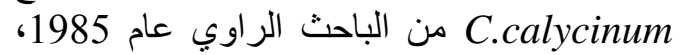

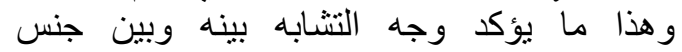
Linaria عhaenorhinum [19] [19. ومن المعلومات المثبتة

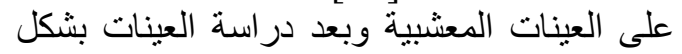

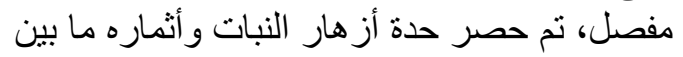

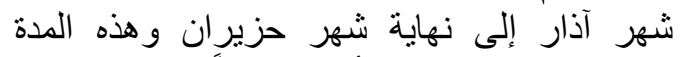

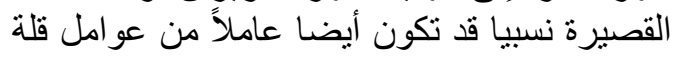
انتشار الجنس في العراق.

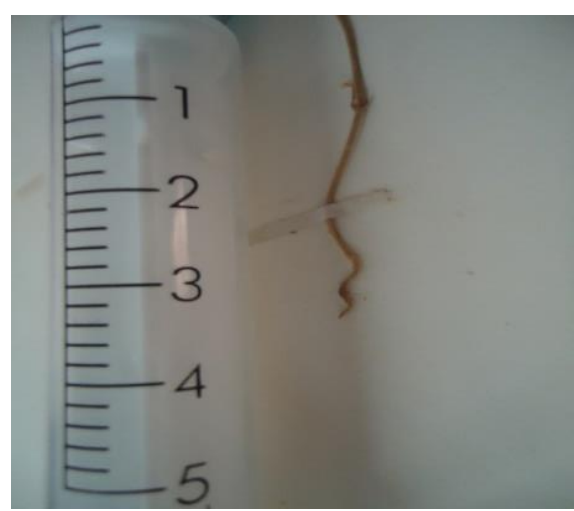

\section{C. rubrifolim}

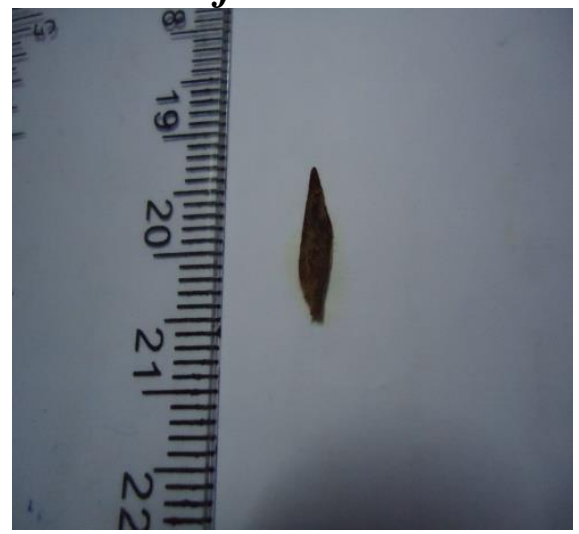

C. rubrifolim
يرجع ذللك الى الجفاف المنتشر في اغلب مناطق

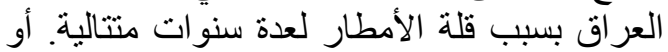
ربما يعود لقلة انتشار الجنس بشكل عام لعام في العراق،





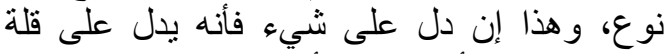

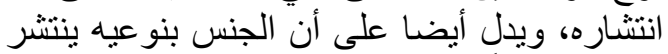



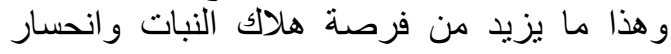

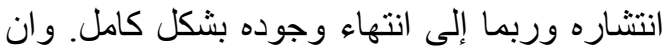

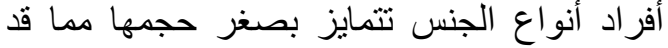

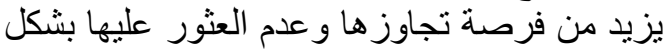
يسير. إلا أن فرصة ازدهار انتشار الجنس ليست

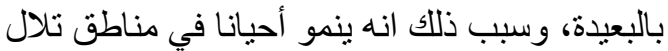







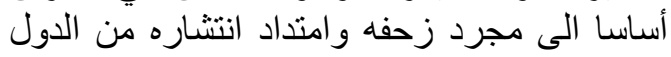
المجاورة مثل إيران وتركيا. ومما تجدر الإشارة



C calycinum.

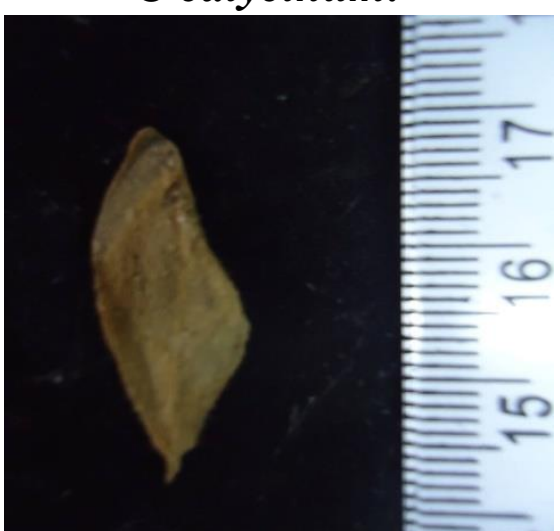

C. calycinum 


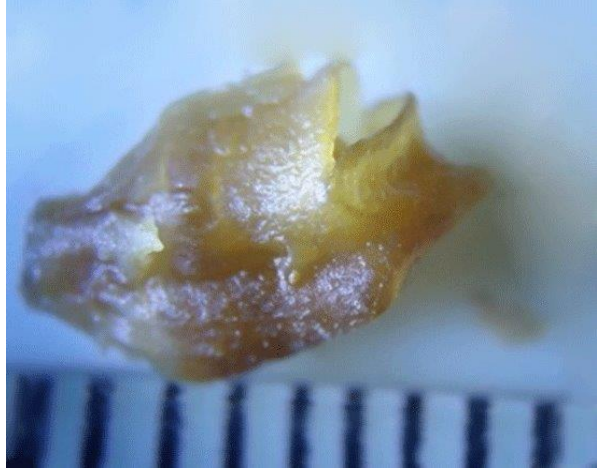

C. rubrifolim



C. rubrifolim
الثمار

البذور



C. calycinum

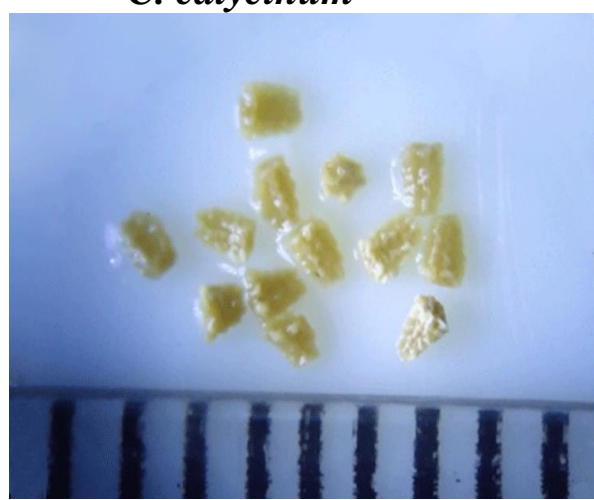

C. calycinum

شكل (1) أثكال وأبعاد الجذور والأوراق والثمار والبذور للنوعين قيد الدراسة.



C. calycinum 


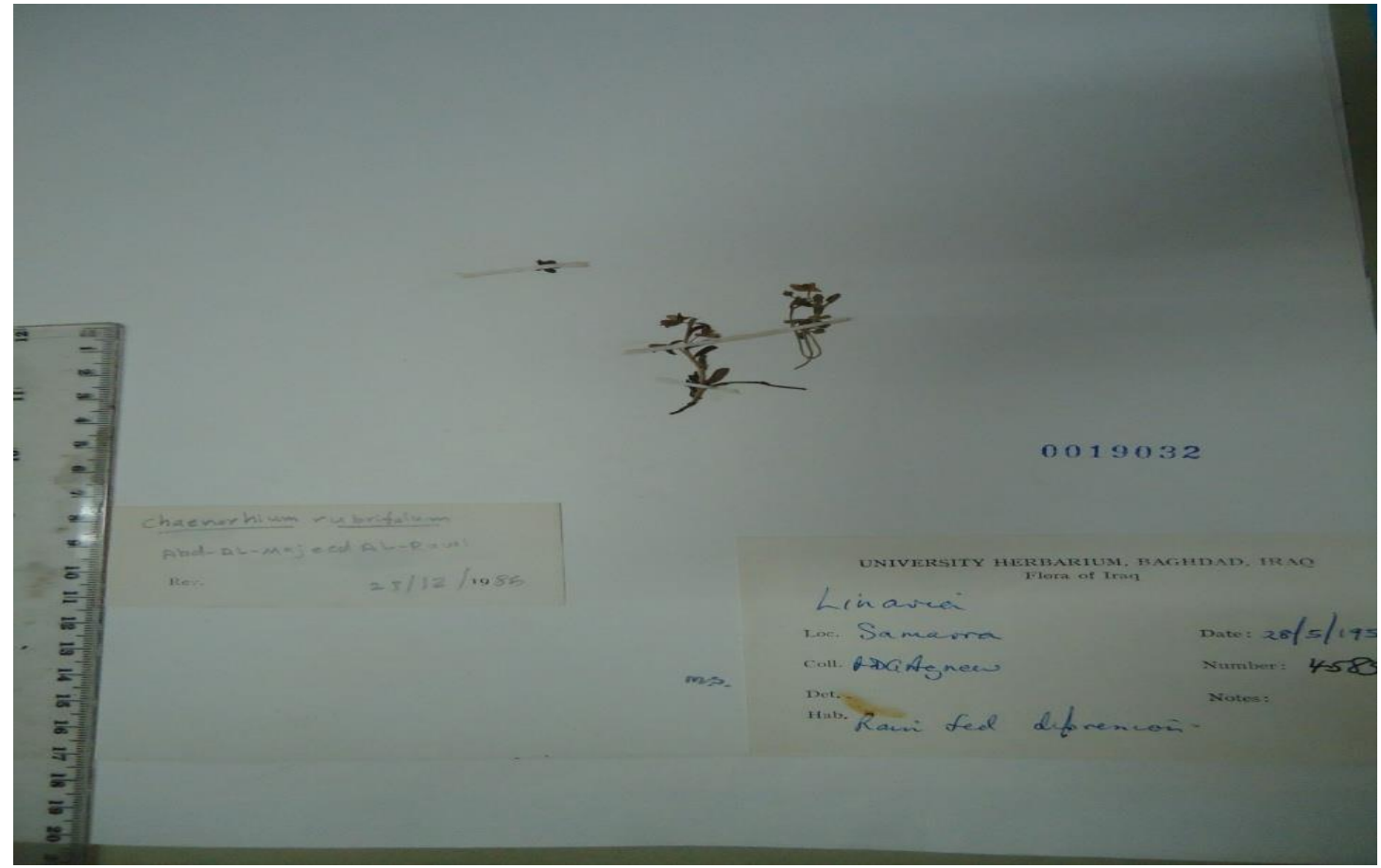

C. rubrifolim

شكل (2) نماذج من العينات المعثبية للنوعين قيد الاراسة.

7 .Boissier, E1879. Flora orienalis. Vol. 2. Genevae et Basileaa, Apud $\mathrm{H}$. Georg, Bibliopolam, Lugduni. 1159 pp.

8. Fischer, 1981. Scorphulariaceae in Flora Iranica No. 147. Akademische Druck-U Verlagsansalt, Austria.

9. Judd, W. S., C. S. Campbell, E. A. Kellogg and P. F. Stevens 1990. Plants systematics. Sinauer associates, Inc., Sunderland, Massachusetts, U.S.A. $464 \mathrm{pp}$.

10. Ridda, Th. J. and W. H. Dawood 1982. Geographical distribution of wild vascular plants of Iraq. National herbarium of Iraq (Unpublished). 140 pp.

11. Rechinger, K.H. 1964 Flora of lowland Iraq. Verlag von J. Cramer. $746 \mathrm{pp}$.

12. Al-Rawi, A. 1964. Wild plants of Iraq with their distribution. Ministry of Agriculture and Irrigation. Gover. Press. 220 pp.

1. Davis, P. H. and V. H. Heywood 1963. Principles of Angiosperms taxonomy. Oliver and Boyd, Edindurgh. 558 pp.

2. Lawrence, G. H. M 1951 . Taxonomy of vascular plants. The Macmillan Company. New York. 823 pp.

3. Richardson, I.B.K. 1978 . Scorphularianceae. In V. H. Heywood, Flowering plants of the worls. Oxford. Univ. press. $335 \mathrm{pp}$.

4. Bentham, G. 1876 . Scrophulariaceae. In: Bentham, G. and J. D. Hooker, Genera plabtarum. Vol. 2 Wheldonand Welsey, LTD. And Verlag J. Cramer.

5. Post, G.E. 1933 Flora of Syria, Palestine and Sina. Vol.2. American Press. Beirut. 255-261. 928 pp.

6. Wettstein, R. V. 1891 Scorphulariaceae. In Engler, A., and K. Prantl. Die Naturlichen Pflanzenfamilien. IV. 3b. Wilhelm Engelman, Leipzig. 
162. Akademische druck - u Verlagsan stalt, Graz - Austria. 555 pp.

21. Guest, E. 1996. Flora of Iraq. Ministry of Agriculture, Republic of Iraq. Vol. 1. $213 \mathrm{pp}$.

22. Stearn, W. T. 1973. Botanical latin. Third edition. David \& Charlos, London. 566 pp.

23. الكاتب، يوسف منصور 1988. تصنيف النباتات

البذرية. الطبعة الأولى. دار الكتب للطباعة الكبة





تصنيف النبات. الطبعة الأولى. دار الكتب

للطباعة و النشر ، جامعة الموصل. 379 3ص.

25. Radford, A. E., W. C. Dikson, J. R. Massey and C. R. Bell 1974 Vascular plants systematics. Harper and Row. $891 \mathrm{pp}$.
13. Zohary, M 1946. The flora of Iraq and its phytogeographical subdivision. Baghdad Government press. 201 pp.

14. Willis, J. C. 1973. A dictionary of the flowering plants and ferns. Eighth edition. Cambridge Univ. press. 1245 pp.

15. Stella, R. C. 1966. British Plants. G. Bell \& Sons LTD. London. `34.

16. Tank, David C. 2006 "Review of the systematic of Scrophulariaceae s.1. and their current disposition". Australian Systematic Botany 19 (4): 289-307. Doi: 10.1071/SB05009.

17. Botanical Journal of the Linnean Society, Volume 81, Issue 3, Article first published online: 28 JUNE 2008.

18. Huxley, A, ed. 1992. New RHS Dictionary of Gardening. ISBN 0-33347494-5.

19. Davis, P. H. 1972. Flora of Turkey and the east Aegean Islands. Vol. 4. Edinburgh, Univ. Press. 657 pp.

20. Rechinger, K.H. 1981. Chaenorhinum In: Rechinger, K. H., Flora Iranica. No.

\title{
Taxonomical and Comparative morphological study for two wild species of the genus Chaenorhinum (D.C.) Reichb. (Scrophulariaceae) in Iraq.
}

\author{
Fatin H.Al-Dulaimi ${ }^{*} \quad$ Ali H.Al-Musawi ${ }^{* *}$ \\ Al-H.Al-Mousawi ${ }^{* * * *}$ \\ * Department of Biology - College of Educatin for Women- Baghdad University. \\ ** Department of Biology- College of Science- Baghdad University. \\ *** Department of Biology- College of Science for Women - Baghdad University
}

\begin{abstract}
:
Comparative morphological study has been treated for two species of the genus Chaenorhinum (D.C.) Richb., These species were:

1. Chaenorhinum calycinum

2. Chaenorhinum rubrifolium (Robill. \& cast. Ex Lam. \& DC.) Fourr.

The genus belong to the family Scorphulariaceae. Morphological characters has been studies for: root, stem, leaves, flowers (calyx, corolla, androcium including filaments and anthers, gynocium including ovary, style and stigma), fruits and seeds also has been characterized. Key for there two species presented using some quantitative characters. Other characters like shape of fruits and seeds were used too, and they were of a useful taxonomic value.
\end{abstract}


